Revista 2020

\title{
Estrategias para la confección y calificación de cuestionarios en la plataforma Moodle*
}

\author{
Daniela Soledad Gonzalez ${ }^{\mathrm{a}}$
}

\begin{abstract}
Resumen: Este artículo surge de la actividad docente en la plataforma Moodle. Su objetivo es ilustrativo, busca dar un ejemplo de diversas situaciones que se produjeron en el uso de los cuestionarios de la plataforma y ofrecer opciones didácticas que pueden resultar de utilidad a la hora de resolver situaciones similares. Se pretenden focalizar dos aspectos del uso de los cuestionarios de Moodle: la confección de las consignas y la revisión manual de los resultados automáticos que arroja la plataforma. En primer lugar, se hace una breve explicación acerca de los cuestionarios: en qué consisten, qué tipos de preguntas permiten realizar, cómo se obtienen los resultados y cómo se revisan. En segundo lugar, se ofrecen algunas herramientas utilizadas en la práctica docente personal para la confección apropiada de las consignas. En tercer lugar, se proporcionan algunas orientaciones sobre el modo de llevar a cabo las revisiones de las calificaciones automáticas de Moodle. Finalmente, se cierra el trabajo con una conclusión que sintetiza lo trabajado.
\end{abstract}

Palabras clave: didáctica; Moodle; cuestionarios; consignas; evaluación.

Fecha de recepción: 5 de febrero de 2020 Fecha de aprobación: 30 de mayo de 2020

Disponible en línea: 22 de octubre de 2020.

Cómo citar: Gonzalez, D. S. (2020). Estrategias para la confección y calificación de cuestionarios en la plataforma de Moodle. Academia y Virtualidad, 13(2), 91-102. https://doi.org/10.18359/ravi.4565

* Artículo de investigación.

a Doctora en letras. Profesora en la Universidad Nacional de Cuyo, Mendoza, Argentina. Correo electrónico: gonzalezdanielasoledad@yahoo.com.ar ORCID: https://orcid.org/0000-0003-2437-531X 


\title{
Strategies for the creation and rating of questionnaires in the Moddle platform
}

\begin{abstract}
This article arises from the teaching activity on the Moodle platform. Its objective is illustrative, it seeks to give an example of various situations that occurred using the platform questionnaires and to offer didactic options that may be useful when solving similar situations. The aim is to focus on two aspects of Moodle questionnaires: preparation of the instructions and the manual review of the automatic results produced by the platform. First, a brief explanation is made about the questionnaires: what they are made up of, what types of questions are allowed to ask, how the results are obtained, and how they are reviewed. Second, some tools used in the face-to-face teaching practice are offered for appropriate preparation of instructions. Third, some guidance is provided on how to carry out automatic Moodle grade reviews. Finally, the paper closes with a conclusion summarizing the work.
\end{abstract}

Keywords: didactics; Moodle; questionnaires; instructions.

\section{Estratégias para a elaboração e correção de questionários na plataforma Moodle}

Resumo: Este artigo surge da atividade docente na plataforma Moodle. Seu objetivo é ilustrativo, pois procura dar um exemplo de diversas situações que se produziram no uso dos questionários da plataforma e oferecer opções didáticas que podem resultar de utilidade na hora de resolver situações similares. Pretende-se focalizar dois aspectos do uso dos questionários do Moodle: a elaboração de enunciados e a revisão manual dos resultados automáticos que a plataforma produz. Em primeiro lugar, faz-se uma breve explicação a respeito dos questionários: em que consistem, que tipos de perguntas permitem realizar, como se obtêm os resultados e como se revisam. Em segundo lugar, oferecem-se algumas ferramentas utilizadas na prática docente pessoal para a elaboração apropriada de enunciados. Em terceiro lugar, proporcionam-se algumas orientações sobre o modo de realizar as revisões das correções automáticas do Moodle. Finalmente, encerra-se o trabalho com uma conclusão que sintetiza o trabalhado.

Palavras-chave: didática; Moodle; questionários; enunciado; avaliação. 


\section{Introducción}

La práctica docente está llamada a acercarse constantemente a los intereses y hábitos de los alumnos. Uno de los hábitos más reconocidos del alumnado actual es su constante interacción con las computadoras y otros dispositivos móviles que les permiten el acceso a la web. En este ámbito, la plataforma Moodle (Modular Object-Oriented Dynamic Learning Enviroment) se ha posicionado desde hace unos años como un ámbito ideal tanto para el aprendizaje autorregulado (Valenzuela-Zambrano y Pérez-Villalobos, 2013) como para el aprendizaje mediado por el profesor (Suárez Guerrero, 2009; Marín Díaz y Maldonado Berea, 2011) ${ }^{1}$ y para el aprendizaje colaborativo (Llorente Cejudo, 2007). Permite el acceso desde una matriz al material elaborado por el profesor y a recursos contenidos en la red (Gómez Rey, Hernández García y Rico García, 2009). Además, posibilita la creación de actividades interactivas, instrumentos de trabajo y actividades de autoevaluación (López, Romero y Ropero, 2010).

Este artículo se origina en la incorporación de las aulas virtuales a la práctica docente. En particular, se trata de la creación de actividades en la plataforma Moodle destinada a la evaluación semanal de la lectura del material de cátedra y los apuntes de clase de la asignatura Morfología y Sintaxis de la Facultad de Filosofía y Letras de la Universidad Nacional de Cuyo durante el ciclo lectivo 2019.

En el programa de la asignatura se indicó que las condiciones de regularidad del espacio curricular eran las de contar con un cierto puntaje correspondiente a las actividades virtuales y otro correspondiente a las instancias presenciales escritas. En cuanto al modo de realización de los cuestionarios, se les indicó que se resolverían una vez por semana, los jueves de 10:00 a. m. a 11:00 a. m. Se les solicitó prestar mucha atención a las consignas para tratar de evitar la mayor cantidad de errores posibles a la hora de la liberación de resultados.

1 Para un panorama sobre las plataformas de enseñanza virtual para usos educativos (qué son, cuáles existen, qué uso se les puede dar, sus ventajas e inconvenientes), Cf. Sánchez Rodríguez (2009) y Domínguez Lázaro (2010). El alcance de este trabajo se ha acotado a una plataforma en específico: Moodle.
Además, como las consignas llevan un nivel alto de estructuración, es con los detalles mínimos con los que se hace la diferencia entre una respuesta correcta y una incorrecta. Se insistió en ello. También se insistió en que observaran el deletreo de sus respuestas y la ortografía de las palabras antes de enviar las respuestas. Respecto de la ortografía, se les indicó que podían consultar con la profesora sus dudas.

Los alumnos podían resolver el cuestionario en el aula con asistencia de la docente o en cualquier otro lugar donde se encontraran que les permitiera el acceso a internet. Podían utilizar sus computadoras personales, pero también teléfonos y otros dispositivos con conexión a internet. Para los que no contaran con la tecnología necesaria, existía la opción de utilizar las computadoras de la sala de informática de la facultad.

Contextualizado el surgimiento de la experiencia que se presentará en este escrito, se pasa, a continuación, a detallar diversas situaciones que se produjeron en dicha experiencia y a ofrecer opciones didácticas que pueden resultar de utilidad a la hora de resolver situaciones similares. Se comienza por explicar en qué consisten los cuestionarios de Moodle y cuál es su utilidad.

\section{Los cuestionarios de Moodle}

La plataforma Moodle ofrece variados recursos. Entre los más útiles se encuentran los cuestionarios. Como se señala en el Curso ABC Moodle (2019), que puede realizarse en la plataforma para aprender a utilizarla, "podemos usarlos [a los cuestionarios] para probar la adquisición de competencias, la asimilación de contenidos, elaborar guías de aprendizaje y crear herramientas de autoevaluación, entre otros usos posibles" (Módulo 2: Actividades y recursos. Sección, actividad cuestionario).

Para crear un cuestionario hay que instalar y configurar el recurso cuestionario en el lugar que se elija del aula virtual y, luego, crear y configurar las preguntas que se van a incluir en él. En cuanto a la configuración del cuestionario, es destacable el hecho de que este instrumento permite seleccionar si se muestra a los alumnos o no con anticipación a su resolución, permite determinar el periodo de tiempo durante el que estará disponible para su 
resolución y hasta la cantidad de intentos que el alumno podrá realizar para su culminación. Inclusive, se puede elegir si, luego de varios intentos, se calificará cada resolución con la nota más alta alcanzada o con un promedio de las notas obtenidas en los diversos intentos.

Otro punto digno de ser enfatizado es que las preguntas que se incorporarán al cuestionario se generan en un banco de preguntas y luego se asignan al cuestionario que se elija. Esta forma de trabajo ofrece versatilidad de opciones, puesto que pueden asignarse las mismas preguntas a diversos cuestionarios y por lo tanto, pueden crearse cuestionarios distintos según las preguntas que se elijan para integrarlos.

Las preguntas que integran los cuestionarios pueden ser de muy diversos tipos. Aquí no se trabajarán todos ellos. Solo se listarán los que se han trabajado, que son los más útiles para el objeto de estudio y metodología propia de la disciplina que se dicta (Morfología y Sintaxis) y para los objetivos de las actividades, que fueron principalmente los de control de lectura y afirmación de conocimientos.

Los tipos de preguntas que se van a desarrollar son los siguientes:

(a) Preguntas de verdadero o falso.

(b) Preguntas de opción múltiple.

(c) Preguntas de completamiento.

Las preguntas de verdadero o falso constan de un enunciado que debe calificarse según los dos parámetros que dan nombre a la pregunta. Un ejemplo de pregunta de verdadero o falso es el siguiente:

\section{Coloque verdadero (V) o falso (F)}

El enfoque funcionalista de los fenómenos lingüísticos postula que la estructura de las lenguas no está condicionada por las funciones que el enunciado cumple en situación comunicativa concreta

\section{Seleccione una: \\ O Verdadero \\ O Falso}

El otro tipo de preguntas que se pueden generar es de completamiento. Su enunciado solicita un concepto que debe responder manualmente el alumno. A continuación, un ejemplo:

¿Qué disciplina lingüística estudia lo siguiente?:

Para formar sustantivos se pueden utilizar, entre otros, los siguientes segmentos fónicos: /-e $\theta a /$, /-ura/, /-al/, /-miento/ y /-ería/.

\section{Respuesta:}

El último tipo de preguntas que resultó de utilidad es el de las preguntas de opción múltiple. En esta clase de consignas se pide al alumno elegir entre varias opciones la correcta. La siguiente es una pregunta de este tipo:

\section{Elija la opción correcta:}

La relación (función) existente entre los planos del contenido y la expresión es de...

\section{Seleccione una:}

- a. Coordinación

O b. Subordinación

○ c. Interdependencia

Una pregunta de opción múltiple un tanto más compleja es la siguiente:

El nivel morfológico es estudiado por la morfología que se encarga de describir cómo se las palabras de una lengua, cómo una misma palabra en el discurso, cuáles son las categorías morfológicas de y cuáles, las unidades necesarias para el análisis morfológico desde la unidad máxima, la vacío morfológica hasta

la mínima, el

En este caso, las opciones para ubicar en los espacios vacíos son las siguientes: flexión, forman, varía, palabra y morfema. Se despliegan todas en el menú que se abre al hacer clic en cada espacio vacío.

Para las preguntas de opción múltiple se pueden colocar tres opciones de respuesta o más. El alumno las visualizará a todas a la vez a la hora de tener que elegir en cada espacio vacío que deba rellenar. El sistema las baraja y las coloca en distinto orden cada vez que presenta la pregunta para dificultar que los alumnos se pasen las respuestas entre sí. 
Además, en la edición de las consignas, se coloca la respuesta esperada, el puntaje que se le asigna a la consigna (en los casos de respuestas libres o con opciones, se puede elegir qué porcentaje del puntaje lleva cada alternativa) y una retroalimentación. La retroalimentación es un mensaje que el docente deja preparado para que haga su aparición cuando el alumno envíe su respuesta, de manera que ya al momento de haberla enviado sepa qué resultado obtuvo. Se pueden plantear retroalimentaciones generales y retroalimentaciones particulares para las respuestas erróneas. A continuación, se puede apreciar un ejemplo de consigna con su retroalimentación:

\section{Complete la siguiente afirmación:}

Los dos planos que distingue Saussure en el signo lingüístico son el plano del

[[1]] y el plano del [[2]]. Hjelmslev opta por denominarlos respectivamente plano del de la [[4]]. [[3]] y plano

\section{Retroalimentación:}

La respuesta correcta es:

\section{Complete la siguiente afirmación:}

Los dos planos que distingue Saussure en el signo lingüístico son el plano del

[significado] y el plano del [significante]. Hjelmslev opta por denominarlos respectivamente plano del y plano de la [contenido] [expresión]

Una vez resuelto el cuestionario, el profesor puede descargar la corrección automática que le otorga la plataforma. Además, puede revisar cada intento de los alumnos por separado y hacer cambios en la puntuación, si lo desea. Las notas pueden descargarse en un archivo en formato Excel. Si se hacen cambios en la puntuación de ciertos alumnos en particular, se puede dar clic en la opción "recalificar todo" y se obtiene un archivo actualizado con todas las notas finales.

El archivo tiene el formato que se ejemplifica a continuación:

Tabla 1. Ejemplo de informe de resultados del cuestionario de Moodle

\begin{tabular}{|c|c|c|c|}
\hline Apellido(s) & Nombre & $\begin{array}{l}\text { Dirección } \\
\text { de correo }\end{array}$ & Estado \\
\hline$X X X$ & $X X X$ & XXX@gmail.com & Finalizado \\
\hline Comenzado & Finalizado & $\begin{array}{l}\text { Tiempo } \\
\text { requerido }\end{array}$ & $\begin{array}{c}\text { Calificación } \\
/ / 10\end{array}$ \\
\hline $\begin{array}{l}4 \text { de abril de } \\
2019 \cdot 10: 00\end{array}$ & $\begin{array}{l}4 \text { de abril de } \\
2019 \cdot 10: 36\end{array}$ & $\begin{array}{c}35 \text { minutos } \\
28 \text { segundos }\end{array}$ & 6 \\
\hline
\end{tabular}

Fuente: elaboración propia.

Los cambios en el otorgamiento del puntaje realizados en las revisiones pueden verse acompañados de comentarios como muestra el siguiente ejemplo:

\section{Complete el siguiente enunciado (con tres palabras): \\ Según el número de morfemas, las palabras se clasifican en Monorfemicas $\boldsymbol{x}$}

La respuesta correcta es monomorfémicas y polimorfémicas.

\section{Comentario:}

Error de deletreo. Prestar atención a ello. Por única vez, se otorga el puntaje.

Figura 1. Comentario del docente a la revisión de la calificación automática de los cuestionarios de Moodle. Fuente: Curso ABC Moodle (2019)

Por otra parte, el sistema otorga un informe numérico al docente de los resultados del grupo de alumnos, como el que sigue:

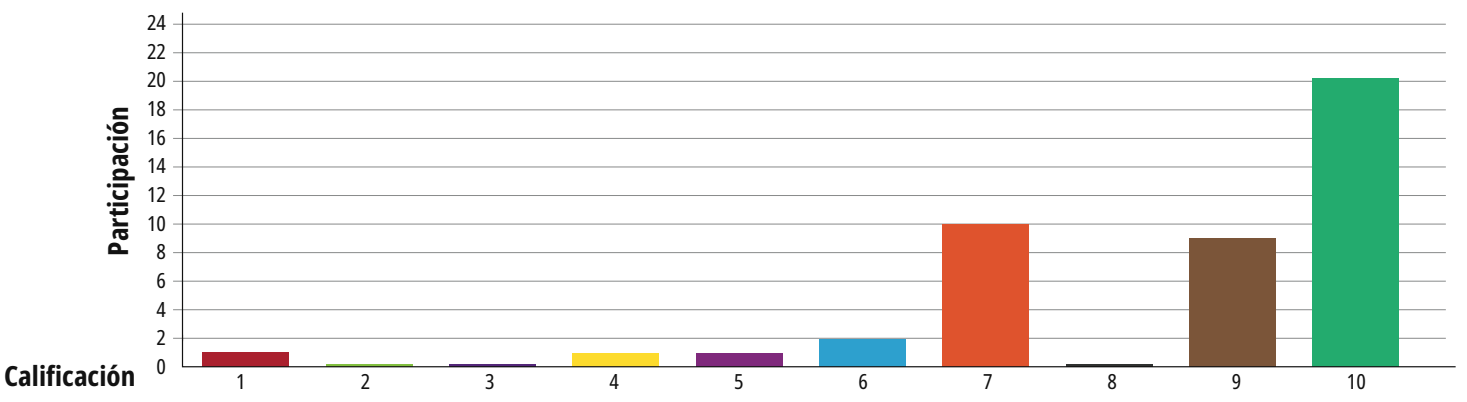

Figura 2. Informe gráfico de resultados de un cuestionario de Moodle.

Fuente: Curso ABC Moodle (2019) 


\section{Aspectos metodológicos para la confección de consignas}

En esta sección se abordará el modo de elaborar actividades para los cuestionarios de Moodle de la forma más didáctica y precisa posible Para las consignas de verdadero o falso o de opción múltiple hay que cuidar la redacción para no dar lugar a ambigüedades de interpretación. Lo interesante de estas consignas es que no dejan lugar a las respuestas abiertas, por lo cual no ofrecen problemas a la hora de hacer la revisión y permiten una redacción capciosa que resulta provechosa en cuanto al ejercicio mental y la transferencia de conocimientos que implica.

Un caso particular: si se desea comprobar que los alumnos comprendieron bien que un enfoque funcionalista de los fenómenos lingüísticos plantea una dependencia de la semántica por parte de la sintaxis (Langacker, 2008), se puede negar esta misma aseveración y, con ello, obtener una pregunta que deben calificar como falsa. Esto se puede apreciar en el siguiente ejemplo:

\section{Coloque verdadero (V) o falso (F)}

No hay autonomía entre la estructura gramatical y las consideraciones semántico-pragmáticas.

Seleccione una:

Verdadero

O Falso

Para comprender el próximo ejemplo hay que tener en cuenta que los morfemas se pueden clasificar según tres criterios: el semántico, el distribucional y el sintáctico (Pena, 1999). El primero de ellos distingue los morfemas léxicos de los derivativos y de los gramaticales. El segundo criterio distingue entre morfemas raíces, prefijos, sufijos, interfijos, etc. Si mezclamos un criterio con las clases de morfemas que distingue el otro, tenemos otra pregunta de verdadero o falso con falso como respuesta correcta.

Distribucionalmente, los morfemas se clasifican en léxicos, derivativos y gramaticales.

Seleccione una:

O Verdadero

O Falso
Se puede generar preguntas capciosas cambiando un adjetivo que modifique a un sector no central del enunciado para evaluar el nivel de atención y minuciosidad en el manejo de los conceptos. La consigna que sigue hace esto por medio del adjetivo distintos:

/kom-l, /ko-/ y /kon-/ son alomorfos de distintos morfemas.

Seleccione una:

O Verdadero

O Falso

En lugar de decir de distintos morfemas debería decir del mismo morfema. La particularidad de los alomorfos es, justamente, que son diversos segmentos fonológicos que expresan un mismo significado (Pena, 1999).

Por supuesto que es fácil generar preguntas con errores para observar si los alumnos los detectan, pero no se debe abusar de este recurso porque pueden resultar preguntas predecibles. Se sugiere redactar una cantidad equivalente de preguntas de verdadero o falso con respuesta correcta verdadero. Para este tipo de preguntas hay que presentarles una inferencia que se puede extraer o una transferencia posible de un conocimiento determinado y no su mera enunciación tal como pueden encontrarla en el texto. Por ejemplo, no conviene enunciar la consigna como "hay morfemas representados siempre con la misma forma fonológica o morfo y, por eso, se denominan morfos únicos" (Pena, 1999, p. 4347; Tamola, 2019, p. 36-37), sino así:

En el caso de los morfos únicos, siempre que aparezca el morfema, aparecerá realizado por el mismo morfo.

\section{Seleccione una:}

O Verdadero

O Falso

Otra posibilidad de presentar información verdadera es la de plantear una aplicación de un conocimiento general. En lugar de enunciar "un morfo amalgama es un segmento fonológico que expresa varias categorías de flexión", se puede confeccionar la siguiente consigna: 
Los morfos de tiempo, modo y aspecto de los verbos son amalgama.

\section{Seleccione una:}

O Verdadero

O Falso

Las consignas de opción múltiple pueden dar lugar a algunas dificultades en la resolución que compliquen la calificación automática si no se tiene en cuenta que el alumno puede elegir una opción para más de un espacio vacío. En el siguiente caso de una actividad con opción múltiple, se puede apreciar cómo la consigna pide al alumno un orden específico en la selección de las alternativas. Ese orden está marcado por el adverbio respectivamente, que, para más ayuda al alumno, ha sido colocado en negrita.

\section{Complete la siguiente afirmación:}

Los dos planos que distingue Saussure en el signo lingüístico son el plano del

$y$ el plano del . Hjelmslev opta por denominarlos respectivamente plano del y plano de la

Otro ejemplo de pregunta de opción múltiple en la que es importante observar el orden en que se pide ubicar las opciones es la siguiente:

\section{Complete la siguiente afirmación:}

La morfología comprende dos partes: la y la _-_._-_. La primera de ellas estudia los procedimientos de _-_ de las palabras, su estructura. La segunda estudia la de las palabras en el uso.

La parte que estudia la cadena de derivación de las palabras es la Morfología

Las opciones de respuestas disponibles son morfología léxica, morfología flexiva, formación, variación, léxica y flexiva. Las respuestas esperadas son, respectivamente: morfología léxica, morfología flexiva, formación, variación, léxica. Un alumno cometió el error de seleccionar como opción final morfología léxica en lugar de léxica. En ese caso, se había predeterminado en la plataforma al editar la pregunta que se le debía asignar el $80 \%$ del puntaje por conocer el concepto que se requería, pero no seguir la consigna, que solicitaba una sola palabra en el espacio final y no dos.

Con las consignas que piden un orden especial de las respuestas, hay que prestar mucha atención al modo en que se enuncian o se arman con vistas a no generar confusiones en los alumnos. Les debe quedar claro qué se espera que se responda en cada lugar. Un ejemplo de consigna con estas precauciones es la siguiente:

Ubique las siguientes características de las relaciones lingüísticas paradigmáticas y sintagmáticas en el cuadro comparativo que se encuentra a continuación siguiendo las instrucciones.

Ubicar en la primera fila dos de las siguientes características: sintagma - en ausencia - selección - paradigma.

Ubicar en la segunda fila dos de las siguientes opciones: sintagma - en ausencia - selección - en presencia.

Ubicar en la tercera fila dos de las siguientes opciones: selección - paradigma - combinación - en presencia.

\begin{tabular}{|c|c|}
\hline $\begin{array}{c}\text { Relaciones lingüísticas } \\
\text { paradigmáticas }\end{array}$ & $\begin{array}{c}\text { Relaciones lingüísticas } \\
\text { sintagmáticas }\end{array}$ \\
\hline & \\
\hline & \\
\hline & \\
\hline
\end{tabular}

La respuesta correcta a este ejercicio es la siguiente y en ella se puede observar cómo solo los pares de palabras correspondientes a cada fila podían ser rescatados de las opciones que se les dieron a los alumnos para ubicar en cada casillero:

\begin{tabular}{|c|c|}
\hline $\begin{array}{c}\text { Relaciones lingüísticas } \\
\text { paradigmáticas }\end{array}$ & $\begin{array}{c}\text { Relaciones lingüísticas } \\
\text { sintagmáticas }\end{array}$ \\
\hline Paradigma & Sintagma \\
\hline En ausencia & En presencia \\
\hline Selección & Combinación \\
\hline
\end{tabular}

Cuando se delinean las preguntas de completamiento, incluso cuando se espera de los alumnos una palabra y siendo esta un término, es decir, un concepto muy específico y marcado por la comunidad discursiva en la que se trabaja es de vital 
importancia tener en cuenta todas las variantes que pueden ocurrírseles a los alumnos como respuestas a las actividades.

Por ejemplo, si esperamos que la respuesta a una consigna sea el principio de articulación (de las lenguas naturales humanas) (Martinet, 1949, 1957), hay que pensar en las variantes a esa redacción de la respuesta que pueden surgir. En el caso particular de la autora de este artículo se proyectaron las que siguen:

- Principio de articulación de las lenguas naturales humanas

- Principio de articulación de las lenguas naturales

- Principio de articulación

- Articulación

- Articulación de las lenguas

- Articulación de las lenguas humanas

- Articulación de las lenguas naturales humanas

- Articulación de la lengua

- Principio de articulación de las lenguas

- Principio de articulación de la lengua

- Principio de articulación de las lenguas humanas

- La doble articulación de las lenguas

- Doble articulación

- La doble articulación de las lenguas

- El principio de doble articulación

- La doble articulación de las lenguas naturales

- Principio de doble articulación

- La doble articulación

- El principio de doble articulación de las lenguas naturales

- Principio de doble articulación de las lenguas naturales

- Lenguas doblemente articuladas

Incluso habiendo predeterminado todas estas variantes, aparecieron redacciones sorpresivas no tenidas en cuenta como "lenguas doblemente articuladas" que hubo que recalificar como respuestas correctas. Otro punto que, por supuesto, hay que dejar establecido en la predeterminación de las respuestas es que se deben tomar como válidas tanto las que estén escritas en mayúsculas como las que estén en minúsculas.

Un caso que no había sido contemplado, por ejemplo, es que los alumnos pusieran punto final a su respuesta. Lo que se hizo al revisar la respuesta fue modificar el puntaje asignado (de cero a uno, esto es, se asignó el puntaje completo) y aclararlo mediante el siguiente comentario: "en las próximas respuestas no coloque el punto final. Esta vez, se le coloca el puntaje completo a esta consigna”. En el siguiente apartado, se podrán observar otros ejemplos de este estilo.

\section{Revisiones de calificaciones automáticas}

Si bien el sistema de calificaciones automáticas está pensado para ahorrar tiempo a los docentes y permitir la autoevaluación de los alumnos, resulta de gran utilidad revisar los resultados de los cuestionarios porque siempre puede haber un desliz en la confección del instrumento. La revisión manual de los resultados permite al docente evaluar si se otorga una parte del puntaje o el puntaje completo en algunos casos particulares. Además, dicha revisión posibilita observar en qué puntos hubo más errores y qué aspectos hay que reforzar en el grupo-clase y con cada alumno en particular. No se debe olvidar que la enseñanza virtual no debe ser una mera interacción persona máquina, sino que debe tender a conformar una "interacción de tipo Persona-Máquina-Persona” (Suárez Guerrero, 2009, p. 1).

Por ejemplo, si hay un error ortográfico (lo cual no es menor, sobre todo en el área de Letras), pero el concepto está claro, se puede decidir otorgar la mitad del puntaje a la consigna. A continuación, se ejemplifican casos en los cuales la revisión de los resultados automáticos llevó a realizar cambios en el puntaje.

\section{Ejemplos de comentarios a las revisiones de consignas:}

En este apartado, se presentan algunos casos reales de consignas en las cuales hubo que hacer cambios 
en los puntajes asignados automáticamente por la plataforma Moodle. Se coloca en primer lugar la consigna; en segundo lugar, la retroalimentación que reciben los alumnos al terminar el llamado "intento" de resolución (i. e., al enviar su respuesta definitiva); finalmente, las respuestas de los alumnos y los comentarios que hizo la profesora al revisar la calificación.

\section{Caso 1}

\section{Consigna:}

¿Qué disciplina lingüística estudia lo siguiente?:

Para formar sustantivos se pueden utilizar, entre otros, los siguientes segmentos fónicos: /-e $\theta a /$, /-ura/, /-al/, /-miento/ y /-ería/.

\section{Retroalimentación:}

La respuesta correcta es: La morfofonología

\section{Comentario del profesor al hacer la revisión:}

A la respuesta "Morfofonológico":

Se preguntaba por la disciplina y no por el nivel lingüístico correspondiente. Se asigna la mitad del puntaje.

\section{A la respuesta "Morfofonologia" [SIC]:}

Morfofonología lleva tilde. Por única vez, se deja pasar el error ortográfico y se otorga el puntaje a la consigna.

\section{Caso 2}

\section{Consigna:}

¿Qué concepto se está definiendo a continuación?

Particularidad de las lenguas naturales humanas por la que los elementos más simples del signo lingüístico se asocian entre sí para permitir que, con un número finito de elementos, se pueda no solo crear un número infinito de componentes lingüísticos y de mensajes, sino también analizar la lengua según diferentes niveles o perspectivas.

\section{Retroalimentación:}

La respuesta correcta es: Principio de articulación de las lenguas naturales humanas.
Comentario del profesor al hacer la revisión:

A la respuesta "Principio de articulación del lenguaje":

El concepto del lenguaje es más amplio que el de lengua. Aquí se pedía identificar una característica de las lenguas naturales humanas, no del lenguaje en general. Se otorga la mitad del puntaje porque el principio en sí fue identificado.

A la respuesta "El principio de articulación":

La respuesta es correcta. Entre las opciones de respuesta predeterminadas no he considerado la enunciación con el artículo inicial. Lo cambio en el cuestionario para evitar este inconveniente a futuro. Le asigno el puntaje al intento.

\section{Caso 3}

\section{Consigna:}

¿Qué disciplina lingüística estudia lo siguiente?:

Para formar sustantivos se pueden utilizar, entre otros, los siguientes segmentos fónicos: /-e $\theta a /$, /-ura/, /-al/, I-miento/ y /-ería/.

\section{Retroalimentación:}

La respuesta correcta es: La morfofonología.

\section{Respuesta:}

Morfofología

\section{Comentario del profesor al hacer la revisión:}

Prestar atención al deletreo de las palabras. Un mal deletreo puede ocasionar que se la respuesta sea considerada incorrecta. Por única vez, se asignó el puntaje completo a esta consigna.

Esta última consigna es aquella en la que más se equivocaron los alumnos, puesto que muchos de ellos deletrearon mal y cometieron faltas de ortografía. Algunos también eligieron el nexo coordinante o en lugar del esperado y. Esta elección de o no se había tenido en cuenta al plantear las respuestas predeterminadas. Como ejemplo de las respuestas dadas a los alumnos, se colocan dos, una en el caso de que ellos no hubieran sido los del error, sino el docente (si bien el conector más apropiado es $y$ y no $o$ ) y otra en el caso de que el error hubiera sido de los alumnos. 


\section{Caso 1:}

La respuesta es correcta. Se le asigna el puntaje completo.

\section{Caso 2:}

Su respuesta ha sido "polimorfémicas y Monomorfémicas”. La próxima vez trate de no colocar espaciados extra ni mayúsculas innecesarias. Como la respuesta es conceptualmente correcta, se le asigna el puntaje completo.

\section{Conclusión}

Como se ha podido apreciar a lo largo de este escrito, la actividad docente exige en la actualidad el uso de plataformas virtuales en el entorno de enseñanza como una herramienta para mejorar el aprendizaje en el ámbito individual, el mediado y el colaborativo. Este artículo comenta dos aspectos en particular del uso de Moodle: la redacción de cuestionarios y su evaluación. Se explica en qué consisten los cuestionarios de Moodle, cómo se configuran, qué tipos de preguntas permiten hacer y cómo se evalúan. De los diversos tipos de preguntas disponibles en la plataforma, se desarrollan tres, que son los que mayor utilidad ofrecen para evaluar la lectura de material bibliográfico y afianzar conocimientos; estos tipos son los siguientes: (a) preguntas de verdadero o falso, (b) preguntas de opción múltiple y (c) preguntas de completamiento.

A lo largo del artículo, se comentan experiencias personales de su autora en orden a ejemplificar las diversas situaciones que se pueden producir en el uso de los cuestionarios de la plataforma y ofrecer estrategias didácticas que puedan resultar de utilidad a la hora de resolver situaciones similares. En el caso de las consignas de verdadero o falso o de opción múltiple, hay que cuidar la redacción para no dar lugar a ambigüedades de interpretación. Esta clase de consignas permite una redacción capciosa, lo cual mide la atención del alumno, además de las inferencias y transferencias de conocimientos que es capaz de hacer.

A la hora de redactar las actividades de verdadero o falso, resulta tentador generar gran cantidad de preguntas con errores para observar si los alumnos los detectan, pero no se debe abusar de este recurso porque pueden resultar predecibles. Se sugiere redactar una cantidad equivalente de preguntas de verdadero o falso con respuesta correcta verdadero. Este último tipo de preguntas debe presentar inferencias o transferencias del conocimiento. En cuanto a la redacción de las preguntas de opción múltiple, hay que dejar muy en claro el orden en el que se desea que se coloquen las alternativas y acotar la información de modo tal que una y solo una de las opciones pueda colocarse en cada espacio vacío. La confección de las consignas de completamiento exige también un detallado acotamiento del ámbito del conocimiento que se aborda y del concepto que se desea como respuesta.

En relación con la revisión manual de los resultados automáticos que arroja el sistema, esta permite al docente evaluar si se otorga puntaje en algunos casos particulares. Además, dicha revisión posibilita observar en qué puntos hubo más errores y qué aspectos hay que reforzar en el grupo-clase y con cada alumno en particular. Las preguntas de verdadero o falso no suelen exigir recalificación. Las de opción múltiple pueden dar lugar a complicaciones si no se ha tenido en cuenta que el alumno podía elegir más de una opción para cada espacio vacío. En cuanto a las preguntas de completamiento, estas son las que más dificultades acarrean a la hora de la corrección automática. Incluso cuando se espera de los alumnos una sola palabra y siendo esta un término o vocablo técnico, es muy difícil que el profesor haya tenido en cuenta todas las variantes que podían ocurrírseles a los alumnos como respuestas, por lo cual se sugiere revisar los resultados, recalificar lo que esté bien respondido y hacer aclaraciones sobre los errores de deletreo, puntuación o redacción que hayan dado como incorrecta la respuesta en la corrección automática.

\section{Referencias}

Aulas Virtuales Facultad de Filosofía y Letras-UN Cuyo. (12 de abril de 2019). Recuperado de: https://www.virtual.ffyl.uncu.edu.ar

Curso ABC Moodle. (12 de abril de 2019). Aulas Virtuales Facultad de Filosofía y Letras-UN Cuyo. Recuperado 
de: https://www.virtual.ffyl.uncu.edu.ar/course/view. php?id=20

Domínguez Lázaro, M. (2010). Moodle, una plataforma formativa con gran proyección en los nuevos modelos de enseñanza. Didáctica, Innovación y Multimedia, (19), 1-14.

Gómez Rey, I., Hernández García, E. y Rico García, M. (2009). Moodle en la enseñanza presencial y mixta del inglés en contextos universitarios. Revista Iberoamericana de Educación a Distancia, 12(1), 169-193.

Langacker, R. (2008). Cognitive Grammar. Nueva York, United States: Oxford University Press. doi: https:// doi.org/10.1093/acprof:oso/9780195331967.001.0001

Llorente Cejudo, M. (2007). Moodle como entorno virtual de formación al alcance de todos. Revista Científica de Comunicación y Educación, XV(28), 197-202. doi: https://doi.org/10.3916/C28-2007-20

López, J., Romero, E., y Ropero, E. (2010). Utilización de Moodle para el desarrollo y evaluación de competencias en los Alumnos. Formación universitaria, 3(3), 45-52. doi: https://doi.org/10.4067/S071850062010000300006

Marín Díaz, V., y Maldonado Berea, G. (2011). El alumno universitario cordobés y la plataforma virtual Moodle. Pixel-Bit. Revista de Medios y Educación, (38), 121-128.
Martinet, A. (1949). La double articulation linguistique. Travaux du Cercle linguistique de Copenhague, 5, 3037. doi: https://doi.org/10.1080/01050206.1949.104162 89

Martinet, A. (1957). Arbitraire linguistique et double articulation. Cahiers Ferdinand de Saussure, (15), 105-116.

Pena, J. (1999). Partes de la morfología. En Demonte, V., y Bosque, I. (Eds.). Gramática descriptiva de la lengua española. Vol. 3. (pp. 4305-4366). Madrid: Espasa.

Sánchez Rodríguez, J. (2009). Plataformas de enseñanza virtual para entornos educativos. Pixel-Bit. Revista de Medios y Educación, (34), 217-233.

Suárez Guerrero, C. (2009). Estructura didáctica virtual para Moodle. Didáctica, Innovación y Multimedia, (13), 1-12.

Tamola, D. (2019). Curso de Morfología del Español en Fichas. Mendoza: Facultad de Filosofía y Letras (Material de uso interno de la cátedra de Morfología y Sintaxis de la FFyL-UN Cuyo).

Valenzuela-Zambrano, B., y Pérez-Villalobos, M. (2013). Aprendizaje autorregulado a través de la plataforma virtual Moodle. Educación y Educadores, 16(1), 66-79. doi: ttps://doi.org/10.5294/edu.2013.16.1.4 
